\title{
Coordination of paediatric oncology care: an explorative Swedish case study
}

\author{
Christian Gadolin \\ University West, Trollhättan, Sweden and \\ Chalmers University of Technology, Gothenburg, Sweden, and \\ Erik Eriksson and Patrik Alexandersson \\ Chalmers University of Technology, Gothenburg, Sweden
}

Cordination of paediatric oncology care

\begin{abstract}
Purpose - The aim of this paper is to empirically describe and analyze factors deemed to be relevant for the successful provision of coordinated paediatric oncology care by physicians and nurses involved.

Design/methodology/approach - A qualitative case study primarily consisting of interviews.

Findings - The paper's findings indicate that certain factors (i.e. distinct mission, clear treatment protocols and support from external stakeholders) relevant for the provision of coordinated paediatric oncology care have not received sufficient attention in previous research. In addition, emphasis is placed on the necessity of facilitating constructive working relationships and a bottom-up perspective when pursuing improved care coordination.

Originality/value - The factors described and analyzed may act as insights for how paediatric oncology might be improved in terms of care coordination and thus facilitate care integration. In addition, the paper's findings identify factors relevant for further empirical studies in order to delineate their generalizability.
\end{abstract}

Keywords Paediatric oncology care, Care coordination, Care integration, Care quality

Paper type Research paper

\section{Introduction}

Survival rates for paediatric cancer patients have dramatically improved in recent decades, often attributed to international collaborative efforts to validate protocol-driven treatments as a viable route towards successful treatment outcomes (Pui et al., 2011; Unguru, 2011). With major strides in strict medical outcomes having been achieved, other effects of the treatment and medical/social rehabilitation for the child, as well as his or her family, were identified at an early stage as important aspects for further study (Bradlyn et al., 1996). Consequently, although medical considerations are and will continue to remain relevant in the provision of improved and adequate care for paediatric oncology patients (Pui et al., 2011), the necessity of paediatric care coordination has become a prominent topic in research as well as in practice (De et al., 2011).

Care coordination should be understood as a "team-driven activity that organizes and drives service integration" (Kuo et al., 2018, p. 224). It has been found to be directly correlated with patients' satisfaction and the perceived quality of the care provided (Matlow et al., 2006). Moreover, failure to provide coordinated care has a substantial impact on the healthcare system's ability to efficiently utilize available resources (Zurynski et al., 2019; Eriksson et al., 2020). In order to develop coordination of care provision, notions related to

(C) Christian Gadolin, Erik Eriksson and Patrik Alexandersson. Published by Emerald Publishing Limited. This article is published under the Creative Commons Attribution (CC BY 4.0) licence. Anyone may reproduce, distribute, translate and create derivative works of this article (for both commercial and non-commercial purposes), subject to full attribution to the original publication and authors. The full terms of this licence may be seen at http://creativecommons.org/licences/by/4.0/legalcode

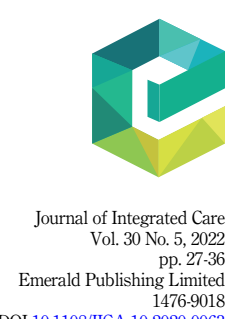

Received 21 October 2020

Revised 8 January 2021 19 February 2021

Accepted 20 February 2021 
JICA

30,5

both general management and organizational theory have been given major influence, however, not with unanimous success (Kaehne, 2019). The substantial reliance of policymakers on top-down policy initiatives and interventions when attempting to increase care coordination produces difficulties in achieving actual change as they rarely have the desired effect on healthcare professionals' practices, often arising from a failure to consider the social context of implementation (Yong and Cameron, 2019). Physicians and, to some extent, nurses are the professional groups which, historically as well as currently, have demonstrated the most difficulties in engaging in improvements in the quality of healthcare in relation to a broader definition of quality, improved care coordination for example, which does not reflect solely their own immediate interaction with the patients (Berwick, 1989; Blumenthal, 1996; Reay and Hinnings, 2009; Arman et al., 2014; Currie and Spyridonidis, 2016; Gadolin, 2017). The unwillingness of physicians and nurses to engage in quality improvements in terms of a broader understanding of quality is often reflected in the fact that healthcare professionals frequently encounter obstructions to care coordination related to communication and collaboration across professional and organizational boundaries (cf. Jolanki et al., 2017; Fraser, 2019).

In reviewing the idea of coordinated care, Ehrlich et al. (2009) argue that the literature is more concerned with providing superficial descriptions of concepts that might facilitate it, such as collaboration and knowledge transfer, while offering "little discussion of the actual practice that might be implemented in order to enact them" (p. 619), a notion seemingly still relevant today (Hughes et al., 2020). Care coordination has been emphasized as vital in counteracting the fragmented care that all children with special healthcare needs face (Cohen et al., 2011; Kuo et al., 2014; Edelstein et al., 2017), along with cancer patients in general (Cortis et al., 2017; Gadolin et al., 2020). Nonetheless, given the major emotional difficulties for family members when a child is diagnosed with cancer, often related to a perceived uncertainty with regard to the medical treatment process (Clarke and Fletcher, 2003; Svavarsdottir, 2005; Björk et al., 2005; Klassen et al., 2008), the necessity of coordinating paediatric oncology care cannot be overstressed (Matlow et al., 2006; Baker et al., 2008; Stark et al., 2016; Kaye et al., 2017).

Consequently, while previous research has established the necessity of coordinating paediatric oncology care, it has been less useful in providing context-specific details in relation to its actual execution. In combination with the notion that physicians' and nurses' involvement is pivotal, but often difficult to attain, in successfully implementing quality improvements in healthcare (e.g. Gadolin and Andersson, 2017; Andersson and Gadolin, 2020), it is necessary to study the factors deemed to be of relevance for these professional groups when providing coordinated paediatric oncology care. In an effort to contribute to such studies, the aim of this paper is to empirically describe and analyze factors deemed to be relevant for the successful provision of coordinated paediatric oncology care by physicians and nurses involved, through the provision of detailed empirical accounts that might act as insights for how to improve paediatric oncology in terms of care coordination and thus facilitate care integration (cf. Kaehne, 2019).

\section{Methods and setting}

Given the nature of the paper's research aim, the study's initial task was to identify a case were the paediatric oncology care provided was deemed to be successful in relation to care coordination. Labelling care as successfully coordinated poses challenges as success may be dependent on a certain perspective or perspectives indicative of success, such as patients' and their families' perceiving the care provided as cohesive. The paediatric cancer care provided by healthcare actors in a region in western Sweden was identified as suitable in relation to these criteria, due to patients' and their families' assessments of the care provided in terms of 
quality and cohesiveness. The regional perspective was chosen due to the fact that complex care needs to be designed in alliance with, and taking into account, multiple actors relevant to the system. Further, it is considered that there is a high level of independence for regional governance in the Swedish healthcare system, and, due to regional sovereignty, the regional level is where practical and strategic adjustments can be made. Moreover, representatives of a regional strategic improvement intervention had identified the paediatric care in the region as a suitable example of how paediatric care could be organized within the whole region due to outstanding results in relation to integration, coordination and efficacy. The region's paediatric oncology care was consequently deemed to be of relevance for this paper's research aim and hence constitutes the case for this study.

Paediatric oncology in the region is structured around the collaboration between seven different hospitals, the largest of which, the university hospital, is situated in the regional capital and houses the region's paediatric oncology centre. It consequently constitutes the hub for collaboration within the region. The university hospital is responsible for updating and implementing research-based treatment protocols, as well as organizing ongoing professional education within the region as a whole. It also houses the region's highly specialized care. Interventions in relation to the treatment of a specific patient that requires highly specialized care are therefore performed here. Moreover, all diagnoses of paediatric cancer are formally performed at the university hospital. The other six county hospitals involved in the collaboration are responsible for all other medical treatment provided for patients within their geographical area after diagnosis. However, healthcare professionals at the county hospitals maintain an intimate collaboration with their colleagues at the university hospital, usually experts within the sub-speciality of paediatric oncology, throughout the medical treatment of each patient. This study focuses broadly on the coordination of the care provided: from the child's first contact with the healthcare system, throughout initial treatment and rehabilitation and also coordination with relevant actors extrinsic to the healthcare system (cf. Eriksson and Hellström, 2020).

Data collection primarily consisted of qualitative interviews, which were deemed highly relevant as they enabled the perceptions and understandings of actors to be examined (Stake, 2010). The interviewees were mainly physicians and nurses as the involvement of these groups of professionals is considered critical but often difficult to obtain in relation to improvements in the quality of healthcare (Berwick, 1989; Blumenthal, 1996; Reay and Hinnings, 2009; Arman et al., 2014; Currie and Spyridonidis, 2016; Gadolin, 2017), but also due to the fact that it was mainly them who had the initial contacts with the patients and their families. The bulk of the interviews were conducted at the university hospital as it housed most of the region's expertise in relation to paediatric oncology. The first interview was carried out with the operations manager (a physician), and the second interview was with the operations manager and the quality improvement worker appointed to the department. These two had previously tried to delineate the success factors perceived by physicians and nurses involved in the collaboration, which was discussed as part of these interviews. The factors provisionally identified were included in the semi-structured interview guide, which enabled the interviewer to steer the conversation towards the phenomena being studied, while simultaneously allowing both the interviewer and the interviewee to elaborate upon relevant topics (Kvale, 2007). All interviews following these two initial interviews started with the open question: "What do you perceive to be the success factors for the coordination of paediatric oncology care?" Once the interviewees had exhausted the success factors that they personally perceived, questions regarding the provisionally identified success factors were asked, provided they had not been addressed organically during the interview, which was the case more often than not. Moreover, following first-level interpretations of the data collected (cf. Alvesson and Sköldberg, 2009), the perceived consensus of success factors identified was discussed on a continuous basis with the informants interviewed at a later stage of the study.

Cordination of paediatric oncology care 
JICA 30,5

In addition to the initial interviews with the operations manager and the quality improvement worker, five interviews were conducted at the university hospital with three physicians and two nurses (who were tasked with facilitating coordination of the care).

In addition, one interview was conducted at each county hospital. As these hospitals were smaller, every physician and nurse at the respective paediatric care unit could potentially be involved in the care of paediatric cancer patients. Interviews were therefore conducted with those with most experience of the phenomenon studied, that is, the physician and the nurse most actively involved in the paediatric oncology care provided at the hospital. These interviews were conducted in pairs so that the study would benefit from two parties being able to discuss success factors from two distinct professional perspectives and thus provide complementary points of view and together provide a more nuanced and richer description of perceived success factors (cf. Morris, 2001; Wilson et al., 2016). A total of six paired interviews were consequently performed, incorporating six physicians and six nurses. The case study comprised a total of 13 interviews, consisting of ten physicians (including the operations manager), eight nurses and one quality improvement worker. All interviews were transcribed verbatim.

Three observations were also carried out as a supplement to the aforementioned interviews. One of them was conducted at a regional meeting to discuss desired improvements to all paediatric care within the region. This meeting focused on how the regional paediatric oncology care could constitute a source of inspiration for other areas of care to improve their operations. The other two observations were conducted at meetings with participants from the seven different hospitals. These meetings were ongoing, taking place once every six months. The observations enabled the interviews to be performed in a more informed fashion as the dynamics for the collaboration were experienced first-hand (cf. Alvesson and Sköldberg, 2009). Moreover, it was possible to further discuss topics of interest identified at these meetings with the interviewees and thus in more detail.

The data were analyzed utilizing a conventional content analysis (Hsieh and Shannon, 2005), with the empirical data first read and re-read on multiple occasions. All data relevant to understanding the factors and prerequisites enabling the coordination of paediatric oncology care were then sorted. In accordance with the paper's aim, further coding of the data sorted was subsequently performed in order to present sub-categories enabling a more comprehensive and detailed description of the success factors identified. The quotes presented are illustrative of these interpretations (cf. Silverman, 2001). The results section below will present the factors identified as relevant for success.

\section{Factors perceived as relevant for success}

\section{Formalized collaboration}

All interviewees affirmed the formalized collaboration between the seven hospitals as fundamental to the success of paediatric oncological care in the region. This formalized collaboration creates a network where parties interact on a regular basis through biannual physical gatherings. This establishes an arena for healthcare professionals engaged in paediatric oncology care from right across the region to socialize and become acquainted with each other. These gatherings - a consequence of the formalized collaboration with the university hospital acting as the hub - enables everyone engaged in the paediatric oncology care process to put a "name and a face" to every other individual involved in it, regardless of whether they are employed at a remote hospital. This socialization process was deemed to be a basic prerequisite for all of the interviewees in enabling them to actively seek advice and support from other healthcare professionals engaged in the paediatric care process. The formalized collaboration of regional paediatric oncology care enabled a network incorporating regular physical gatherings of everyone engaged in care provision, acting as 
a "springboard" for further conversations and learning among all employees involved in the regional paediatric oncology care.

The regularity of this contact led to the representatives of each hospital acknowledging a joint responsibility for each patient, further enabling an open and constructive dialogue between all parties. As a physician (P1) put it when asked to reflect upon the difference between the collaboration within paediatric oncology care and the collaboration with other departments/wards at the university hospital:

Many of us are reluctant to call other departments at the university hospital to ask for medical advice. I have personally been met on many occasions with scepticism and an unpleasant attitude when I have done so. Of course, that severely obstructs collaboration. The contact within the paediatric care collaboration is characterized by the opposite. We all feel like we are colleagues, despite us working at different places.

\section{Secondments at the university hospital}

Physicians and nurses at the county hospitals are able, and encouraged, to take secondments at the university hospital for a period of at least three months. Most of them have already done so, and personnel at both the university hospital and the other hospitals emphasize that it has greatly increased their ability to - collectively - provide coordinated care through facilitating a range of positive effects. First and foremost, the healthcare professional involved in the secondment is able to expand his or her knowledge and experience surrounding the care and treatment of children with cancer through learning and discussions with the region's leading experts within the field. Furthermore, and even more substantively than the regular regional meetings, the secondments provide the opportunity to build stronger collegial and personal relationships with employees at the university hospital, which by extension makes it easier for the individual on secondment to engage in further contact in order to discuss patients and their treatments. In addition, employees returning from secondments are able to disseminate new insights and knowledge at their own hospital that they have acquired through interaction with experts within the field. In summarizing the benefits of secondment, one of the nurses (N1) at a county hospital stated that:

The most obvious benefit is that we get to know each other, which makes our future collaboration that much easier and smoother. For instance, we know who to call and they know who is calling. I think that is the cornerstone for coordinating the care we provide.

\section{Distinct missions}

Another success factor that many of the interviewees reflected upon was the influence of distinct missions in relation to the collaboration between the seven hospitals. Although representatives from each hospital noted a mutual dependency between the seven hospitals, and especially between the university hospital and the respective hospitals within the collaboration, a congruent and accepted division of labour also exists between them. It entails the university hospital having the mission to diagnose and initiate treatment for all new cancer patients. Unless requiring specific expertise, subsequent treatment of the cancer, as well as secondary infections and illnesses, is undertaken by the respective county hospital. The interviewees deemed that clarity in relation to both mission and main responsibilities reduced misunderstandings and miscommunication between all employees involved in care provision, and thus facilitated coordination.

\section{Clear treatment protocols}

Perceived clarity in relation to treatment protocols was closely linked to the aforementioned success factor. A specific treatment plan is in place for every major paediatric cancer diagnosis. As these plans are perceived to be legitimate by the healthcare professionals
Cordination of paediatric oncology care 
JICA

30,5

involved, they are followed meticulously and thus used to standardize the treatment given to each patient. The clarity of the treatment protocols, as well as their acceptance and concomitant compliance with them, ensures that everyone involved in care provision knows what measures to take, when and by whom. Moreover, it facilitates the provision of information to both the patient and family members regarding the care process. The physicians and nurses involved in care provision believe that formulation of the treatment protocols was made possible in part due to the relatively short and intensive course of the disease. Paediatric cancer is described by the interviewees as a form of dichotomy, "it either goes well or it goes badly", as one of the physicians (P2) put it. This shared sense of urgency facilitates standardization of the care provided, as it is perceived as central in ensuring the chance of a favourable outcome. Another aspect strengthening the professionals' confidence in standardized treatment procedures is the relatively limited influx of new patients. "There is no room for personal interpretations" is the reasoning provided by one of the nurses (N2) interviewed - the best available research and practical knowledge must be applied in order to ensure the patient's well-being and the outcome of the treatment.

\section{Teamwork}

Well-functioning teamwork at each hospital is also highlighted by the interviewees as a prerequisite for facilitating coordination of the care provided. Many of them emphasized their feeling that there is genuine encouragement from all professionals in the team, and that every member of the team should be actively engaged in the provision of care. Analogously, there is no perception of professional hierarchies hindering or inhibiting the teamwork - which the interviewees often feel to be the case in other healthcare contexts. The fundamental reason for this is emphasized as the dense and intensive treatment for children with cancer as it bonds the members of the team together and makes teamwork central in delivering the best care during the treatment. Moreover, several of the interviewees testified that they enjoy good social relationships with colleagues outside of work, which is highly likely to influence the prospects of cooperation within the team. Another aspect that is perceived to weld the team together, and thus facilitate the provision of coordinated care, is the team's shared view of patients and their families. The close and intense contact with them during treatment creates relationships that "go beyond" what many of the interviewees perceive to be common in other care provision processes. In describing the team's perception of the patient, one of the physicians (P3) interviewed stated that "we view our patients differently. We do not even call them patients, we call them children." Moreover, the relationship that forms between the team, the patient and relatives induces a sense of security as they get to know each individual member of the team and hence know which of them is suitable for direct queries.

\section{Support from external stakeholders}

Many of the prerequisites for the successful coordination of regional paediatric care were perceived as having been made possible due to the funding and support of external actors, the Swedish Childhood Cancer Fund being the most prominent, with its main contribution being the monetary resources it provides for the internal and external elements of paediatric cancer care, as well as putting the focus on paediatric cancer care as an important phenomenon in society. The monetary contributions enable the hospitals involved in the collaboration to undertake certain actions and activities that would otherwise not have been possible as they are not included in the regional financing. For instance, they finance salaries for physicians and nurses in different parts of the system, mainly in order to cover vacancies for staff on secondment at the university hospital. Moreover, funding has made it possible to hire nurses with the focus on integration of services outside the hospital setting. This is mainly arranged in the form of participation in schools and other social situations, through explaining and normalizing the child's challenges and answering questions from children, other adults and 
teachers. Most physicians and nurses interviewed accentuated both the importance of external funding in enabling finance of activities central to care coordination and the fact that it facilitated a more holistic approach to the care provided. When speaking about the necessity of taking a holistic approach towards the coordination of the care provided and the facilitatory role of external funding, the operations manager stated that:

The sick child is a part of a family, a part of a class in a school, a member of a football team, and a member of society. Cancer affects all these situations and relationships. We need to be aware of that and counteract the potentially negative impact that the cancer might inflict at all levels of the social paediatric oncology care plane for the child.

\section{Concluding discussion}

In describing and analyzing the success factors that physicians and nurses deemed to be relevant for the successful coordination of paediatric oncology care provided by a region in western Sweden, this paper provides empirical accounts that may be complementary to previous research that has established that clinical networks are vital when seeking to achieve successful care coordination and its associated benefits, though often highlighting the that fact that, despite being vital, it is difficult to engage clinical staff (e.g. Greene et al., 2009; Ferlie et al., 2013; Brown et al., 2016). While some of the factors described, such as teamwork and the necessity of collaboration, have been highlighted previously as fundamental underpinnings for care coordination (Kuo et al., 2018), research has also emphasized the major difficulties in achieving them in a healthcare setting (Gadolin and Wikström, 2016). The empirical descriptions of these factors provided by physicians and nurses may consequently provide further information on how they might be achieved in paediatric oncology care.

The other factors described in this paper - the importance of distinct missions, clear treatment protocols and support from external stakeholders - have not attracted the same attention within the literature which seeks to delineate factors necessary for clinical networks and care coordination. Whereas clear treatment protocols have been identified as relevant for the successful treatment of paediatric cancer (Pui et al., 2011; Unguru, 2011), this paper highlights its potentially interrelated effect on the coordination of care provision. Moreover, given the seeming ambiguity in identifying distinct missions as a relevant factor, as successful collaboration is often founded on allowing the collaborators to influence the work undertaken (Eriksson et al., 2020), we argue that this factor should be studied in greater detail in order to outline how it influences the ability to coordinate paediatric oncology care. The finding that support from external stakeholders is pivotal in executing certain actions and activities is problematic as it suggests that coordination of care is contingent on the benevolence of parties separate from its financiers. Efforts aiming to increase coordination in paediatric care should thus ensure that funding for all activities and actions identified as relevant is secured in order to safeguard its feasibility. Further research is encouraged in other contexts in order to delineate whether these factors are unique to the case studied or if they are indicative of factors relevant for the coordination of paediatric oncology care in general.

Overall, the findings of this paper support the notion that a bottom-up perspective is beneficial in ensuring the coordination of care (cf. Kaehene, 2019), as many of the initiatives vital for it are the result of the active engagement of the healthcare professionals involved in care provision, and that constructive working relationships are pivotal in pursuing all kinds of quality improvements contingent on collaboration (cf. Gadolin and Andersson, 2017). The factors identified in the paper also highlight the fact that, regardless of context, research with the aim of studying success factors in care coordination must also include the lived experiences of the healthcare professionals engaged in its provision. Without such inclusion, there is a risk that important aspects vital to understand the holistic nature of successful care coordination within a particular context are left unelucidated. Moreover, the paper also underlines the fact that coordination is vital in all sequences of care provision; from the child's 
JICA 30,5

initial contact with the healthcare systems, throughout treatment and rehabilitation, and also in relation to other parts of the child's life outside the hospital setting. As this study focuses on the perceptions of physicians and nurses, future studies could potentially explore success factors perceived by actors who are more involved in the later stages of care provision; given that this paper highlights the fact that the factors facilitating care coordination have to be adapted to how the cancer is perceived to be affecting the child, as well as the child's family, at each specific point in time.

In order to further understand factors relevant for successful care coordination from the perspectives of the child and the child's family, as well as to facilitate what is frequently the general aim of an increased focus on the "customer" in the delivery of and improvements to public services (e.g. Needham, 2006; Osborne et al., 2021), further studies could also potentially focus on these narratives in outlining additional factors. Such studies could complement the literature focussing on clinical networks by highlighting actors/ organizations that - from the perspectives of the child and the child's family - might be relevant for the successful coordination of paediatric oncology care and thus enhance the holistic nature of contemporary public service delivery (cf. Osborne et al., 2013).

\section{References}

Alvesson, M. and Sköldberg, K. (2009), Reflexive Methodology: New Vistas for Qualitative Research, SAGE Publications, London.

Andersson, T. and Gadolin, C. (2020), "Understanding institutional work through social interaction in highly institutionalized settings: lessons from public healthcare organizations”, Scandinavian Journal of Management, Vol. 36 No. 22, 101107.

Arman, R., Liff, R. and Wikström, E. (2014), "The hierarchization of competing logics in psychiatric care in Sweden”, Scandinavian Journal of Management, Vol. 30 No. 3, pp. 282-291.

Baker, J.N., Hinds, P.S., Spunt, S.L., Barfield, R.C., Allen, C., Powell, B.C., Andersson, L.H. and Kane, J.R. (2008), "Integration of palliative care practices into the ongoing care of children with cancer: individualized care planning and coordination", Pediatric Clinics of North America, Vol. 55 No. 1, pp. 223-250.

Berwick, D.M. (1989), "Continuous improvement as an ideal in health care”, New England Journal of Medicine, Vol. 320 No. 1, pp. 53-56.

Björk, M., Wiebe, T. and Hallström, I. (2005), "Striving to survive: families' lived experiences when a child is diagnosed with cancer", Journal of Pediatric Oncology Nursing, Vol. 22 No. 5, pp. 265-275.

Blumenthal, D. (1996), "Quality of care - what is it?", New England Journal of Medicine, Vol. 335 No. 12 , pp. 891-894.

Bradlyn, A.S., Ritchey, A.K., Harris, C.V., Moore, I.M., O’Brien, R.T., Parsons, S.K., Patterson, K. and Pollock, B.H. (1996), "Quality of life research in pediatric oncology: research methods and barriers", Cancer: Interdisciplinary International Journal of the American Cancer Society, Vol. 78 No. 6, pp. 1333-1339.

Brown, B.B., Patel, C., McInnes, E., Mays, N., Young, J. and Haines, M. (2016), “The effectiveness of clinical networks in improving quality of care and patient outcomes: a systematic review of quantitative and qualitative studies", BMC Health Services Research, Vol. 16, A360.

Clarke, J.N. and Fletcher, P. (2003), "Communication issues faced by parents who have a child diagnosed with cancer”, Journal of Pediatric Oncology Nursing, Vol. 20 No. 4, pp. 175-191.

Cohen, E., Kuo, D.Z., Agrawal, R., Berry, J.G., Bhagat, S.K., Simon, T.D. and Srivastava, R. (2011), "Children with medical complexity: an emerging population for clinical and research initiatives", Pediatrics, Vol. 127 No. 3, pp. 529-538.

Cortis, L.J., Ward, P.R., McKinnon, R.A. and Koczwara, B. (2017), "Integrated care in cancer: what is it, how is it used and where are the gaps? A textual narrative literature synthesis", European Journal of Cancer Care, Vol. 26 No. 4, e12689. 
Currie, G. and Spyridonidis, D. (2016), "Interpretation of multiple institutional logics on the ground: actors' position, their agency and situational constraints in professionalized contexts", Organization Studies, Vol. 37 No. 1, pp. 77-97.

De, P., Ellison, L.F., Barr, R.D., Semenciw, R., Marrett, L.D., Weir, H.K., Dryer, D. and Grunfeld, E. (2011), "Canadian adolescents and young adults with cancer: opportunity to improve coordination and level of care", Canadian Medical Association Journal, Vol. 183 No. 3, pp. E187-E194.

Edelstein, H., Schippke, J., Sheffe, S. and Kingsnorth, S. (2017), "Children with medical complexity: a

Cordination of paediatric oncology care scoping review of interventions to support caregiver stress", Child: Care, Health and Development, Vol. 43 No. 3, pp. 323-333.

Ehrlich, C., Kendall, E., Muenchberger, H. and Armstrong, K. (2009), "Coordinated care: what does that really mean?", Health and Social Care in the Community, Vol. 17 No. 6, pp. 619-627.

Eriksson, E., Andersson, T., Hellström, A., Gadolin, C. and Lifvergren, S. (2020), "Collaborative public management: coordinated value propositions among public service organizations", Public Management Review, Vol. 22 No. 6, pp. 791-812.

Eriksson, E. and Hellström, A. (2020), "Multi-actor resource integration: a service approach in public management", British Journal of Management. Forthcoming. doi: 10.1111/1467-8551.12414.

Ferlie, E., Fitzgerald, L., McGivern, G., Dopson, S. and Bennett, C. (2013), Making Wicked Problems Governable? The Case of Managed Networks in Health Care, Oxford University Press, Oxford.

Fraser, M.W. (2019), "Elephant in the room: inter-professional barriers to integration between health and social care staff", Journal of Integrated Care, Vol. 27 No. 1, pp. 64-72.

Gadolin, C. (2017), "The logics of healthcare - in quality improvement work", Doctoral dissertation, University of Gothenburg, Gothenburg.

Gadolin, C. and Andersson, T. (2017), "Healthcare quality improvement work: a professional employee perspective", International Journal of Health Care Quality Assurance, Vol. 30 No. 5, pp. 410-423.

Gadolin, C., Andersson, T., Eriksson, E. and Hellström, A. (2020), "Providing healthcare through "value shops': impact on professional fulfilment for physicians and nurses", International Journal of Health Governance, Vol. 25 No. 2, pp. 127-136.

Gadolin, C. and Wikström, E. (2016), "Organising healthcare with multi-professional teams: activity coordination as a logistical flow", Scandinavian Journal of Public Administration, Vol. 20 No. 4, pp. 53-72.

Greene, A., Pagliari, C., Cunningham, S., Donnan, P., Evans, J., Emslie-Smith, A., Morris, A. and Guthrie, B. (2009), "Do managed clinical networks improve quality of diabetes care? Evidence from a retrospective mixed methods evaluation”, BMJ Quality and Safety, Vol. 18 No. 6, pp. 456-461.

Hsieh, H.F. and Shannon, S.E. (2005), "Three approaches to qualitative content analysis", Qualitative Health Research, Vol. 15 No. 9, pp. 1277-1288.

Hughes, G., Shaw, S.E. and Greenhalgh, T. (2020), "Rethinking integrated care: a systematic hermeneutic review of the literature on integrated care strategies and concepts", The Milbank Quarterly, Vol. 98 No. 2, pp. 446-492.

Jolanki, O., Tynkkynen, L.K. and Sinervo, T. (2017), "Professionals' views on integrated care", Journal of Integrated Care, Vol. 25 No. 4, pp. 247-255.

Kaehne, A. (2019), "How organisation theory may help us understand integrated care", Journal of Integrated Care, Vol. 27 No. 1, pp. 2-4.

Kaye, E.C., Snaman, J.M. and Baker, J.N. (2017), "Pediatric palliative oncology: bridging silos of care through an embedded model", Journal of Clinical Oncology, Vol. 35 No. 24, pp. 2740-2744.

Klassen, A.F., Klaassen, R., Dix, D., Pritchard, S., Yanofsky, R., O’Donnell, M., Scott, A. and Sung, L. (2008), "Impact of caring for a child with cancer on parents' health-related quality of life", Journal of Clinical Oncology, Vol. 26 No. 36, pp. 5884-5889. 
JICA 30,5

Kuo, D.Z., Goudie, A., Cohen, E., Houtrow, A., Agrawal, R., Carle, A.C. and Wells, N. (2014), "Inequities in health care needs for children with medical complexity", Health Affairs, Vol. 33 No. 12, pp. 2190-2198.

Kuo, D.Z., McAllister, J.W., Rossignol, L., Turchi, R.M. and Stille, C.J. (2018), "Care coordination for children with medical complexity: whose care is it, anyway?", Pediatrics, Vol. 141 No. Supplement 3, pp. S224-S232.

Kvale, S. (2007), Doing Interviews, SAGE Publications, Thousand Oaks.

Matlow, A.G., Wright, J.G., Zimmerman, B., Thomson, K. and Valente, M. (2006), "How can the principles of complexity science be applied to improve the coordination of care for complex pediatric patients?", BMJ Quality and Safety, Vol. 15 No. 2, pp. 85-88.

Morris, S.M. (2001), "Joint and individual interviewing in the context of cancer", Qualitative Health Research, Vol. 11 No. 4, pp. 553-567.

Needham, C.E. (2006), "Customer care and the public service ethos", Public Administration, Vol. 84 No. 4, pp. 845-860.

Osborne, S.P., Nasi, G. and Powell, M. (2021), "Beyond co-production: value creation and public services", Public Administration, Forthcoming. doi: 10.1111/padm.12718.

Osborne, S.P., Radnor, Z. and Nasi, G. (2013), "A new theory for public service management? Toward a (public) service-dominant approach", The American Review of Public Administration, Vol. 43 No. 2, pp. 135-158.

Pui, C.H., Gajjar, A.J., Kane, J.R., Qaddoumi, I.A. and Pappo, A.S. (2011), "Challenging issues in pediatric oncology", Nature Reviews Clinical Oncology, Vol. 8 No. 9, pp. 540-549.

Reay, T. and Hinings, C.R. (2009), "Managing the rivalry of competing institutional logics", Organization Studies, Vol. 30 No. 6, pp. 629-652.

Silverman, D. (2001), Interpreting Qualitative Data, SAGE Publications, London.

Stake, R. (2010), Qualitative Research: Studying How Things Work, The Guildford Press, New York.

Stark, D., Bielack, S., Brugieres, L., Dirksen, U., Duarte, X., Dunn, S., Erdelyi, D.J., Grew, T., Hjorth, L., Jazbec, J. and Kabickova, E. (2016), "Teenagers and young adults with cancer in Europe: from national programmes to a European integrated coordinated project", European Journal of Cancer Care, Vol. 25 No. 3, pp. 419-427.

Svavarsdottir, E.K. (2005), "Caring for a child with cancer: a longitudinal perspective", Journal of Advanced Nursing, Vol. 50 No. 2, pp. 153-161.

Unguru, Y. (2011), "The successful integration of research and care: how pediatric oncology became the subspecialty in which research defines the standard of care", Pediatric Blood and Cancer, Vol. 56 No. 7, pp. 1019-1025.

Wilson, A.D., Onwuegbuzie, A.J. and Manning, L.P. (2016), "Using paired depth interviews to collect qualitative data", Qualitative Report, Vol. 21 No. 9, pp. 1549-1573.

Yong, L.M.O. and Cameron, A. (2019), "What is the relevance of policy transfer and policy translation in integrated care development?", Journal of Integrated Care, Vol. 27 No. 1, pp. 5-14.

Zurynski, Y., Breen, C., Altman, L., Woolfenden, S. and Ging, J. (2019), "Care coordination for children with medical complexity results in savings for the healthcare system and for families", International Journal of Integrated Care, Vol. 19 No. Supplement 3, A615.

\section{Corresponding author}

Christian Gadolin can be contacted at: christian.gadolin@hv.se

For instructions on how to order reprints of this article, please visit our website:

www.emeraldgrouppublishing.com/licensing/reprints.htm

Or contact us for further details: permissions@emeraldinsight.com 